ARTÍCULOS ORIGINALES

Rev Chil Salud Pública 2019,

Vol 23(1): $42-48$

\section{GARANTÍA EXPLÍCITA EN SALUD ORAL EN ADULTO DE 60 AÑOS: IMPACTO EN LA PERCEPCIÓN DE LA CALIDAD DE VIDA}

\author{
EXPLICIT ORAL HEALTH GUARANTEE FOR ADULTS 60 YEARS OF \\ AGE: IMPACT ON THEIR PERCEPTION OF QUALITY OF LIFE.
}

Patricia Moya Facultad de Odontología, Universidad Finis Terrae;

Santiago, Chile

Escuela de Salud Pública,

Universidad Mayor,

Santiago, Chile

Juan Carlos Caro, Óscar Asmurú,

Paulina Gómez

Facultad de Odontología,

Universidad Finis Terrae;

Santiago, Chile

Lorena Hoffmeister

Escuela de Salud Pública,

Universidad Mayor,

Santiago, Chile.

Patricio González c.

Cirujano-Dentista, CESFAM

Vitacura.

Artículo recibido el 30/04/2018.

Aceptado el 15/03/2019

Financiación

Sin financiación externa.

Conflictos de intereses.

Los autores declaran que no presentan conflicto de intereses.

\section{RESUMEN}

Objetivo: Evaluar la Garantía Explícita en Salud Oral en el Adulto de 60 años (GES-60 años) desde la percepción de la calidad de vida. Método: Se realizó una evaluación antes y después de la prestación del programa GES-60, sin grupo control. Se utilizó el cuestionario Geriatric Oral Health Assessment Index (GOHAl) para cuantificar los cambios en la percepción de la calidad de vida como un indicador de resultado del programa. Se aplicó el test no paramétrico de Wilcoxon para datos emparejados en STATA 12.0. Resultados: Participaron 103 adultos en el estudio y 85,5\% fueron mujeres. Al término del programa GES-60 se presentaron mejoras significativas en la valoración global de la percepción de la calidad de vida relacionada con salud oral, como también en cada una de las dimensiones, particularmente en términos de la limitación psicológica. Conclusión: Existe una significativa contribución del programa en el mejoramiento de la percepción de la calidad de vida de la población intervenida.

Palabras clave: Evaluación, Salud Pública, Calidad de vida, Salud oral, Adulto mayor.

\section{ABSTRACT}

Objective: To evaluate the Explicit Guarantee in Oral Health for Adults 60 years of Age (CES60) on the basis of their perception of quality of life. Method: Evaluations were conducted before and after users participated in the GES-60 program, without a control group. The Geriatric Oral Health Assessment Index (GOHAl) was used to quantify changes in the perception of quality of life as an indicator of the program's results. The nonparametric Wilcoxon test was used to analyze matched data in STATA 12.0. Results: A total of 103 adults participated in the study, and $85.5 \%$ were women. At the end of the GES-60 program, there were significant improvements in the overall assessment of the participants' perception of quality of life related to oral health, in each of the dimensions, particularly in terms of psychological limitations. Conclusion: The program significantly improves the target population's perception of quality of life.

Keywords: Evaluation, Public health, Quality of life, Oral health, Older Adults 


\section{INTRODUCCIÓN}

Chile cuenta con programas ministeriales dirigidos a 80 problemas priorizados de salud denominados Garantías Explícitas en Salud (GES), implementados con la Reforma de salud en el año 2005. ${ }^{1}$ Su principal objetivo está orientado a garantizar el acceso, oportunidad, protección financiera y calidad de la atención de salud al individuo que lo solicita. En este grupo de garantías explícitas se encuentra la "Salud oral integral del adulto de 60 años" (GES-60 años), población objetivo que presenta un marcado deterioro de su salud bucal. ${ }^{2}$

La "Salud oral integral del adulto de 60 años" consiste en garantizar la atención odontológica integral por un cirujano dentista, según necesidad. Está orientada a educar, prevenir, restaurar y rehabilitar la salud bucal del adulto de 60 años y su inicio debe ser antes de los 90 días de realizada la solicitud. La garantía termina cuando se han realizado las actividades que el individuo requería. ${ }^{2}$

El GES-60 años plantea como objetivo contribuir a mejorar la calidad de vida del adulto, como respuesta a la mala salud bucal adquirida durante su vida por no haber recibido tratamientos adecuados y oportunos. ${ }^{2}$ Su puesta en marcha ha significado grandes esfuerzos para el país, tanto en recursos humanos, físicos como económicos, ${ }^{1}$ por lo que resulta relevante conocer en qué grado esta estrategia está efectivamente contribuyendo al logro de uno de los objetivos sanitario del Ministerio de Salud: "Enfrentar los desafíos derivados del envejecimiento de la población"

Hasta ahora, la evaluación del GES-60 años, a nivel de los centros de atención primaria, se ha focalizado en contrastar las actividades programadas versus actividades cumplidas, mientras que a nivel nacional la evaluación se enmarca en la cobertura alcanzada. En la red pública, en el año 2013, la cobertura fue de $32 \%$ mostrando una tendencia decreciente. ${ }^{3}$ La evaluación de los resultados sanitarios de este programa no ha sido abordada sistemáticamente y los estudios son escasos y se limitan a medir el estado de salud bucal y la necesidad de tratamiento. Este tipo de evaluación requiere un abordaje distinto y de la construcción de indicadores afines y de otras fuentes de información que deben ser consideradas en la gestión de la intervención. ${ }^{4}$ La calidad de vida se ha establecido como un indicador de impacto sanitario de enfermedad y suele utilizarse para la evaluación de la eficacia de un tratamiento. ${ }^{5}$

La Organización Mundial de la Salud (OMS) define la calidad de vida como la percepción individual de la posición en la vida en el contexto de la cultura y sistema de valores en el cual se vive y su relación con las metas, expectativas, estándares e intereses (OMS, 2007). Es un concepto amplio, mutidimensional, que considera a la salud oral como un factor que puede afectar las actividades de la vida diaria de la persona, como comunicarse, comer o relacionarse. Una disminución en la autoestima y el bienestar por trastornos en la cavidad bucal dio lugar al concepto de Calidad de Vida Relacionada con Salud Oral (CVRSO) definida como "el bienestar físico, psicológico y social en relación con el estado de la dentición, así como de tejidos duros y blandos de la cavidad bucal".?

Existen diversos instrumentos para medir la CVRSO, entre ellos el Geriatric Oral Health Assessment Index (GOHAI), instrumento validado para el habla hispana que define la salud oral como ausencia de dolor e infección compatible con una dentición funcional y cómoda que permita al individuo continuar en su rol social. Su amplia utilización en adultos mayores ha dejado en evidencia una directa relación entre baja auto percepción de calidad de vida y un pobre estado de salud bucal. ${ }^{6,7}$

Este estudio tiene como objetivo evaluar la Garantía Explícita en Salud Oral en el adulto de 60 años (GES-60 años) desde la percepción de la calidad de vida relacionada con salud oral.

\section{MATERIALES Y MÉTODOS}

Se realizó un estudio de intervención antes-después, sin grupo control, indicado para la evaluación de programas de amplia cobertura o políticas públicas. $^{4}$

Población: La población de estudio estuvo constituida por la totalidad de los pacientes ingresados al GES 60 años (387) en el Centro de Salud Familiar (CESFAM) de la comuna de Vitacura durante el periodo 2014 a 2017. La muestra de trabajo se obtuvo mediante un muestreo por conveniencia, proporcional al total del ingresos por año. Fueron adultos, de ambos sexos, de 60 años de edad que, previa firma de consentimiento informado, ingresaron en forma voluntaria al programa GES-60 años en el centro de 
salud durante los años 2014 al 2017. Fueron incluidos en el estudio aquellos adultos que solicitaron atención odontológica integral mientras tenían 60 años de edad, con necesidad de rehabilitación protésica parcial o total y respondieron la totalidad de las preguntas del cuestionario GOHAI, antes y un mes después de finalizada la atención odontológica. La muestra final quedo constituida por 103 adultos de 60 años.

La rehabilitación protésica del programa GES 60 años se basa en la confección de prótesis dentales removibles, parciales o totales, ya sea para reemplazar un tratamiento previo en malas condiciones o para sustituir inicialmente las piezas dentarias perdidas por caries o enfermedad periodontal. El aparato protésico es realizado en el centro de atención primaria por un odontólogo que posee la especialidad en Rehabilitación Oral. La atención del especialista termina una vez que el paciente ha recibido el alta, por lo tanto sólo se evalúa el éxito o fracaso de éste en el corto plazo (un mes).

Fuente de información. Se utilizó el cuestionario Geriatric Oral Health Assesment Index (GOHAl) para medir percepción de la calidad de vida relacionada con salud oral. Es un instrumento sensible para detectar influencia de los tratamientos dentales en la percepción del paciente sobre su salud oral. Es auto administrado, consta de 12 preguntas con respuesta en escala tipo Likert y valoriza tres dimensiones: función física, función psicosocial y dolor e incomodidad. ${ }^{6-7}$ La percepción de la calidad de vida se calcula como el puntaje obtenido de la sumatoria simple de las respuestas para cada sujeto, dando un rango entre 0 y 60.

Se define "mala calidad de vida" con una puntuación igual o menor a 50; "calidad de vida regular" con puntuación entre 51 y 56, y "buena calidad de vida" con puntuación igual o superior a 57 según el autor. ${ }^{3} \mathrm{~A}$ los efectos de este estudio se crearon categorías de calidad de vida negativa y positiva, definiendo "calidad de vida negativa" con una puntuación igual o inferior a 57 y "calidad de vida positiva" con puntuación mayor a 57 según lo reportado por otros autores 8,9 .

Variables del estudio. Para caracterizar la población estudiada, el cuestionario consideró el auto reporte de variables sociodemográficas: sexo, nivel educacional y variables clínicas: presencia de enfermedades crónicas (Hipertensión Arterial, Diabe- tes Mellitus tipo 2 o ambas), percepción de necesidad de prótesis dental. Se consideró el número de dientes perdidos registrado en el odontograma de la ficha utilizada por el especialista para la rehabilitación con prótesis dental.

Análisis estadístico. Las variables cualitativas fueron presentadas en forma de frecuencias absolutas y porcentajes, mientras que las variables cuantitativas mediante medias y desviaciones estándar, y medianas y rangos intercuartílicos. La comparación antes-después, tanto para el valor global como para cada una de las dimensiones del GOHAI se realizó a través del test no paramétrico de Wilcoxon indicado para muestras relacionadas. Previamente, se testeó la normalidad de la distribución con el test Kolmogorov-Smirnof. Se calculó el valor absoluto y porcentual de cambio en la mediana de las puntuaciones, junto al porcentaje de participantes que mejoran su percepción de la calidad de vida. ${ }^{10}$ Se relacionó la valoración global del GOHAI con cada una de las variables estudiadas y se estableció el nivel de la significancia estadística, valor $\mathrm{p}$ menor a 0,05. Se utilizó el software estadístico STATA 12.0.

\section{RESULTADOS}

Características de la población estudiada. Un 85,5\% de los participantes fueron mujeres y un $44,7 \%$ refiere tener educación básica completa. Un 65,1\% tiene enfermedades crónicas como Hipertensión Arterial (HTA), Diabetes Mellitus tipo 2 (DM) o ambas. La media de dientes perdidos por cualquier causa fue de 13,2 (DE: 7,94) y un 58,2\% presentó pérdida de dientes igual o mayor a 10. El total de participantes refiere necesidad de rehabilitación protésica y más de la mitad $(59,2 \%)$ prótesis dental en ambas arcadas (ver Tabla 1).

Evaluación de los resultados de percepción de calidad de vida. Un 97,1\% de los adultos calificó como mala su calidad de vida relacionada con salud oral al ingreso al programa, cifra que descendió a $34,95 \%$ una vez cumplida la garantía $(p<0,05)$.

Se observaron cambios significativos en la puntuación global del GOHAI, comparando antes y después de la intervención por GES-60 años. El valor de la mediana inicial fue de 35 puntos y final de 53 puntos, como se observa en la Figura 1.

El valor porcentual del cambio de la puntuación global del GOHAI antes y después de la interven- 
Figura 1. Distribución puntaje GOHAI y después de GES- 60 años.

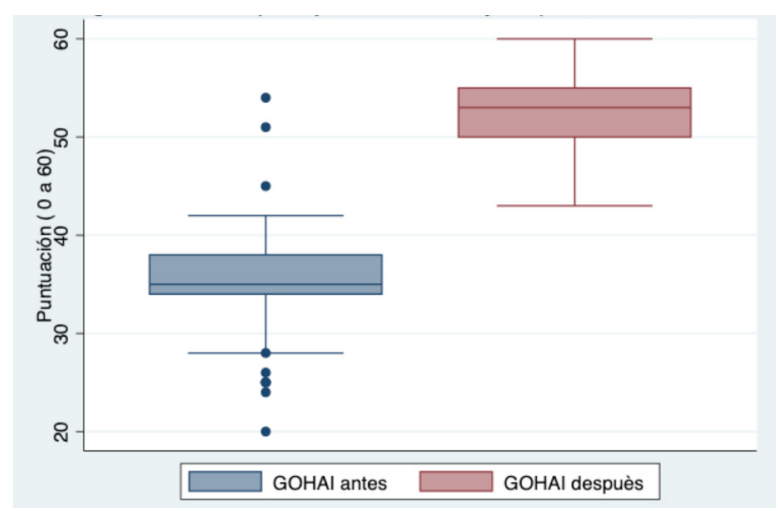

Tabla 1. Características sociodemográficas y clínicas de los adultos de 60 años ingresados por GES en atención primaria de salud.

\begin{tabular}{llll}
\hline Variables & & $\mathbf{n}$ & $\mathbf{\%}$ \\
\hline Sexo & Masculino & 15 & 14,5 \\
\cline { 2 - 4 } & Femenino & 88 & 85,5
\end{tabular}

\begin{tabular}{llll}
\hline Nivel educacional & Educación básica & 46 & 44,7 \\
\cline { 2 - 4 } & Educación media & 23 & 22,3 \\
\hline & Educación superior & 34 & 33,0
\end{tabular}

\begin{tabular}{llcc}
\hline $\begin{array}{l}\text { Presencia de patología } \\
\text { crónica }\end{array}$ & No & 36 & 34,9 \\
\cline { 2 - 4 } & Si & 67 & 65,1
\end{tabular}

\begin{tabular}{llcc}
\hline $\begin{array}{l}\text { Ausencia de dientes } \\
>10\end{array}$ & No & 43 & 41,8 \\
\cline { 2 - 4 } & & & \\
\cline { 2 - 3 } & Si & 60 & 58,2
\end{tabular}

\begin{tabular}{llll}
\hline $\begin{array}{l}\text { Necesidad de rehabili- } \\
\text { tación protésica }\end{array}$ & Arcada superior & 15 & 14,6 \\
\cline { 2 - 4 } & Arcada inferior & 27 & 26,2 \\
\cline { 2 - 4 } & Ambas arcadas & 61 & 59,2 \\
\hline
\end{tabular}

Elaboración propia. ción fue de 51,4\% como se muestra en la Tabla 2. Un 99\% de los participantes mejoró su percepción de calidad de vida.

La dimensión "función física", la cual influye en el comer, hablar y deglutir fue la que generó menor impacto en los adultos de 60 años, después del programa GES, (28,6\% de porcentaje de cambio) afectando a un $71,8 \%$ de los participantes. La dimensión "función psicosocial", que simboliza la preocupación por su salud oral, insatisfacción con la apariencia, autoconciencia acerca de la salud oral y dificultad en el contacto social debido a problemas orales presentó una variación de cambio del $80 \%$, afectando al 99\% de los participantes. Este resultado es similar en la dimensión "dolor e incomodidad".

Puntuación de la percepción de CVRSO y factores sociodemográficos y clínicos. La Tabla 3 muestra las variaciones ocurridas en la puntuación del GOHAl antes y después del programa GES-60 años por las variables estudiadas. La percepción de CVRSO en los adultos antes del GES-60 años fue similar entre hombres y mujeres y entre quienes perciben la necesidad de rehabilitación protésica en una o ambas arcadas. Sin embargo fue levemente mayor en aquellos con nivel educacional básico, en los que no presentan patología crónica y en los que tienen pérdida de menos de 10 dientes en sus arcadas. Estas diferencias no fueron estadísticamente significativas ( $p>0,05)$.

La puntuación del GOHAl después del programa GES-60 años fue mayor para todas las variables estudiadas al comparar con la puntuación registrada antes del GES-60 años. Después del GES 60 años, la puntuación fue levemente mayor en las mujeres de 60 años, en aquellos que refieren nivel educacional básico, con ausencia mayor o igual a 10 dientes y necesidad prótesis en una arcada. Sin embargo, las diferencias observadas no son estadísticamente significativas. La condición de no presentar patología crónica mostró una puntuación más alta que aqueIlos que si refieren presentar patología crónica, siendo esta diferencias estadísticamente significativa. 
Tabla 2.- Cambios en la percepción de la calidad de vida relacionada con salud oral para la valoración global y por dimensiones, antes y después de la intervención por GES-60 años. (Md= mediana).

\begin{tabular}{|c|c|c|c|c|c|c|c|}
\hline \multirow[t]{2}{*}{$\begin{array}{l}\text { GOHAI } \\
\text { Dimensiones }\end{array}$} & \multirow[t]{2}{*}{$\mathbf{n}$} & \multicolumn{2}{|l|}{ Antes } & \multicolumn{2}{|l|}{ Después } & \multirow{2}{*}{$\begin{array}{l}\text { Diferencias } \\
\text { medianas(a) } \\
(\%)\end{array}$} & \multirow{2}{*}{$\begin{array}{l}\text { Participantes que } \\
\text { mejoran(b) } \\
(\%)\end{array}$} \\
\hline & & $\begin{array}{l}\text { Mediana } \\
(\mathrm{Md})\end{array}$ & $\begin{array}{l}\text { Percentil } \\
25-75 \\
\end{array}$ & $\begin{array}{l}\text { Mediana } \\
(\mathrm{Md})\end{array}$ & $\begin{array}{l}\text { Percentil } \\
25-75 \\
\end{array}$ & & \\
\hline $\begin{array}{l}\text { Limitación } \\
\text { Funcional (20 puntos) }\end{array}$ & 103 & 14 & $13-16$ & 18(c) & $15-19$ & 28,6 & 71,8 \\
\hline Limitación psicológica (30 puntos) & 103 & 15 & $14-17$ & $27(\mathrm{c})$ & $24-28$ & 80,0 & 99,0 \\
\hline Dolor e Incomodidad (10 puntos) & 103 & 5 & $5-6$ & $9(\mathrm{c})$ & $8-10$ & 80,0 & 97,1 \\
\hline Valoración global GOHAI (60 puntos) & 103 & 35,0 & $34-38$ & $53,0(\mathrm{c})$ & $50-55$ & 51,4 & 99,0 \\
\hline
\end{tabular}

(a) (Md antes - Md después) /Md antes x 100

(b) Porcentaje de adultos en que la Md después fue mayor a la Md antes. Test de Wilcoxon para datos emparejados

(c) $\mathrm{p}<0,05$

Tabla 3. Puntuación GOHAI en adultos de 60 años antes-después del GES según características sociodemográficas y clínicas.

\begin{tabular}{|c|c|c|c|c|c|c|c|}
\hline \multirow[t]{3}{*}{ Variables } & & \multirow[b]{3}{*}{ media } & \multicolumn{2}{|c|}{ Puntuación cuestionario } & \multicolumn{3}{|l|}{ GOHAI } \\
\hline & & & \multicolumn{2}{|l|}{ Antes } & \multicolumn{3}{|c|}{ Después } \\
\hline & & & DE & $p$ value & media & DE & $p$ value \\
\hline \multirow[t]{2}{*}{ Sexo } & Masculino & 35,46 & 1,95 & 0,51 & 50,86 & 4,94 & 0,09 \\
\hline & Femenino & 35,52 & 5,00 & & 52,30 & 3,79 & \\
\hline \multirow[t]{3}{*}{ Nivel educacional } & Ed. básica & 36,30 & 5,10 & 0,07 & 52,86 & 3,60 & 0,309 \\
\hline & Ed. media & 35,04 & 5,14 & & 50,78 & 4,71 & \\
\hline & Ed. superior & 34,51 & 3,57 & & 51,94 & 3,81 & \\
\hline \multirow[t]{2}{*}{ Presencia de patología crónica } & No & 36,05 & 5,25 & 0,803 & 53,05 & 3,79 & $0,036^{*}$ \\
\hline & $\mathrm{Si}$ & 35,22 & 4,34 & & 51,58 & 4,06 & \\
\hline \multirow[t]{2}{*}{$\begin{array}{l}\text { Ausencia } \\
\text { de dientes }>10\end{array}$} & No & 36,13 & 3,34 & 0,873 & 51,95 & 3,81 & 0,620 \\
\hline & $\mathrm{Si}$ & 35,06 & 5,41 & & 52,20 & 4,13 & \\
\hline \multirow[t]{2}{*}{$\begin{array}{l}\text { Necesidad de rehabilitación } \\
\text { protésica }\end{array}$} & Un maxilar & 35,42 & 4,47 & 0,438 & 52,47 & 3,98 & 0,213 \\
\hline & $\begin{array}{l}\text { Ambos maxi- } \\
\text { lares }\end{array}$ & 35,57 & 4,84 & & 51,83 & 4,00 & \\
\hline Total & 35,51 & 4,67 & & 52,09 & 3,98 & $0,001^{*}$ & \\
\hline
\end{tabular}

Test Student $\left({ }^{*}\right) \mathrm{p}<0,05$ 


\section{DISCUSIÓN}

Existe una creciente necesidad por evaluar el impacto de los programas en Salud Pública, ya sea para la definición de nuevos programas o reformulación de los ya existentes. Sin embargo, por distintas razones (limitaciones económicas, escasa cultura de evaluación entre gestores), son escasos los programas de salud que son evaluados. ${ }^{10}$

Es indudable que, si el GES-60 años tiene por objetivo mejorar la calidad de vida del adulto de 60 años y no sólo limitar las enfermedades bucodentales, integrar indicadores sobre mediciones de la calidad de vida en la historia clínica constituye una evidencia necesaria y relevante para evaluar su impacto en la población beneficiaria. ${ }^{11,12}$

El GOHAl es uno de los instrumentos más utilizados para medir percepción de calidad de vida relacionada con salud oral porque muestra un alto nivel de consistencia interna y confiabilidad (alpha de Cronbach's $>0,79$ ) en todas las edades. Reporta problemas de salud oral relacionados con aspectos fisiológicos, físicos y psicológicos, dando mayor peso a limitaciones funcionales y de malestar o dolor que son inmediatos. ${ }^{13,14}$

Al término del programa GES-60 años, los adultos de 60 años estudiados aquí presentaron mejoras significativas en la valoración global de la percepción de la calidad de vida relacionada con salud oral, como también en cada una de las tres dimensiones medidas por el GOHAI.

De acuerdo a los resultados del presente estudio, hubo diferencias estadísticamente significativas entre las puntuaciones de la percepción de la calidad de vida antes y después de la intervención del GES60 años, corroborando una mejoría en la calidad de vida, mayor en la dimensión psicológica que, por cierto, mejoró al 99\% de los participantes después del programa. Este resultado concuerda con lo señalado por otros autores, donde la función psicosocial sería la reportada con mayor frecuencia de cambio, en especial la relacionada con la incomodidad de comer frente a otras personas por problemas con sus dientes. ${ }^{14-16}$

En relación con las caracteristicas sociodemográficas, algunos autores ${ }^{8,19,20}$ coinciden en que las mujeres tienden a tener una peor percepción de su calidad de vida relacionada con la salud oral en comparación con los hombres y a medida que au- menta la edad, aumenta esta percepción negativa. Si bien en el presente estudio las mujeres presentaron una mejor percepción de CVRSO, las diferencias en la puntuación del GOHAl con los hombres no fueron estadísticamente significativas.

Es importante destacar que después de la intervención por el programa GES-60 años, se observó una mejor percepción de CVRSO en ambos sexos; esto indica que el tratamiento odontológico recibido provoca cambios inmediatos en la calidad de vida de los adultos, independiente del sexo, lo que es concordante con lo referido por otros autores. ${ }^{18}$

Al relacionar la percepción de la calidad de vida con la presencia o ausencia de enfermedades crónicas, se encontró una mayor puntuación del GOHAI en quienes no refieren dichas patologías (Hipertensión Arterial, Diabetes Miellitus tipo 2 o ambas), con significación estadística. Esto concuerda con lo referido por Díaz et al. ${ }^{16}$, que uno de los factores relacionados con el detrimento en la calidad de vida relacionada con salud oral es el antecedente de hipertensión arterial.

La ausencia de dientes y la necesidad de prótesis dental tiene relación directa con la salud del individuo y con una peor percepción de la calidad de vida relacionada con salud oral. Esto obedece a que afecta su función masticatoria con posibles trastornos en la nutrición y muchas veces dolor e incomodidad frente a otras personas al momento de realizar la alimentación. Al ingreso de los adultos al programa, no se observaron diferencias estadísticamente significativas entre la valoración de la CVR$\mathrm{SO}$ y las variables clínicas como ausencia mayor o igual a 10 dientes, junto con la necesidad de una o dos prótesis dentales, dejando de manifiesto que el mayor daño que presentan en su boca no afecta su percepción de la calidad de vida que ya se encuentra deteriorada. La evidencia refiere que quienes necesitan prótesis dentales tienen puntuación más bajas en el GOHAl que quienes no las necesitan, situación similar a la observada en este estudio; los adultos que necesitaban una o dos prótesis dentales tuvieron una puntuación más baja antes de recibir el tratamiento protésico que después de la rehabilitación protésica ${ }^{8,16}$.

Una de las limitaciones del estudio tiene relación con el diseño empleado, ya que al comparar los resultados antes y después de la intervención, sin utilizar grupo control no se puede asumir que 
los cambios ocurridos se deban exclusivamente a la intervención por GES. Sin embargo, ante la imposibilidad de contar con grupo control, se asume que la población permanece "igual" respecto a otros factores que pudieran modificar los resultados y el cambio observado se puede atribuir a la intervención. ${ }^{4,17}$

Es importante considerar que uno de los sesgos más frecuentes en este tipo de estudios es el sesgo de obsequiosidad y que es propio de los participantes que responden las preguntas de un cuestionario o de una entrevista con lo que creen que el investigador considera correcto o quiere escuchar. Esto puede controlarse con la administración auto reportada del cuestionario en una instancia que no sienta la influencia del investigador o entrevistador, como se realizó en este estudio.

Este estudio permite concluir que existe una significativa contribución del programa GES-60 años en el mejoramiento de la percepción de la calidad de vida de la población intervenida.

\section{REFERENCIAS BIBLIOGRÁFICAS}

1. Valenzuela J. Buscando un mejoramiento en la salud oral de los adultos mayores: ¿Es necesaria una reforma al GES-60 años? Rev Chil Salud Pública 2015; 19 (2):181-7.

2. Chile. Ministerio de Salud. Guía clínica Salud Oral Integral para Adultos de 60 años en línea]. Santiago: MINSAL; 2011. Disponible en: http://www.redsalud. gov.cl/archivos/guiasges/saludoral60.pdf

3. Quezada C, Muñoz D, Cueto A, Barraza A. Reforma de Salud en Chile: Evaluación Garantía Explícita en Salud Oral en Población de 60 Años. Int. J. Odontostomat. 2013; 7( 2 ): 319-26.

4. López M, Dell' Olmo M, Perez A, Nebot M. Diseños evaluativos en salud pública: aspectos metodológicos. Gac Sanit. 2011; 25: 9-16.

5. Urzúa M Alfonso. Calidad de vida relacionada con la salud: Elementos conceptuales. Rev. méd. Chile. 2010 ; 138( 3 ): 358-65.

6. Dolan TA, Peek CW, Stuck AE, Beck JC . Threee year changes in global oral health rating by elderly dentate adults. Community Dent Oral Epidemiol. 1998; 26:62-9.

7. Misrachi C, Espinoza I. Utilidad de las Mediciones de la Calidad de Vida Relacionada con la Salud. Rev dent Chile. 2005; 96 (2): 28-35.
8. Moya P, Chappuzeau E, Caro JC, Monsalves MJ. Situación de salud oral y calidad de vida de los adultos mayores. Rev Estomatol Herediana. 2012; 22 (4): 197-202.

9. Durán-Napólitano D, Moya P, Aubert-Valderrama J, Becerra A, Lara-Luer A, Monsalves MJ. Percepción de salud bucal en adultos mayores en dos comunas con desarrollos extremos en Chile. CES Odont. 2016; 29(1)5-12.

10. Villalbí J, Tresserras R. Evaluación de políticas y planes de salud. Gac Sanit. 2011;25 (Supl 1):17-24.

11. Naranjo M. Evaluación de programas de salud. Comunidad y Salud. 2006; 4 (2): 34-7.

12. Lozano J, Saavedra RM, Fernández N. La evaluación del impacto de los resultados científicos. Metodologías y niveles de análisis. Rev Hum Med. 2011; 11(1): 99-117.

13. Deshmukh SP, Radke UM. Translation and validation of the Hindi version of the Geriatric Oral Health Assessment Index. Gerodontology. 2012;29(2):1052-8.

14. Dable R, Nazirkar G, Singh S, Wasnik P. Assessment of Oral Health Related Quality of Life Among Completely Edentulous Patients in Western India by Using GOHAI. J Clin Diagn Res. 2013;7(9):2063-7.

15. Esquivel R, Jiménez J. Efecto de la utilización de prótesis dentales en la percepción de salud bucal. ADM. 2012; 69 (2):69-75.

16. Díaz S, Arrieta K, Ramos K. Impacto de la salud oral en la calidad de vida de adultos mayores. Rev Clin Med Fam. 2012; 5 (1):9-16.

17. Ramos P, Ramosa P, Vázquez N, Pasarín M, Artazcoz L. Evaluación de un programa piloto promotor de habilidades parentales desde una perspectiva de salud pública. Gac Sanit. 2016;30(1):37-42.

18. Bellamy C, Moreno A. Relación entre calidad de vida relacionada con la salud oral, pérdida dental y prótesis removible en adultos mayores de 50 años derechohabientes del IMSS. Av Odontoestomatol. 2014;30(4):195-203.

19. De la Fuente-Hernández J, Sumano-Moreno O, Sifuentes-Valenzuela M, Zelocuatecatl-Aguilar A. Impacto de la salud bucal en la calidad de vida de adultos mayores demandantes de atención dental. Univ. Odontol. 2010; 29(63):83-92.

20. Esquivel R, Jimenez J. Necesidades de Atención Odontológica en Adultos Mayores Mediante la Aplicación del GOHAI. Revista ADM. 2010; 57: 127-31. 